\title{
Gastrointestinal histoplasmosis: a case series from a non-endemic region in North India
}

\author{
Harshal S Mandavdhare, Jimil Shah, Kaushal K Prasad, Roshan Agarwala, Vikas Suri, Savita Kumari, Usha Dutta, \\ Vishal Sharma
}

Department of Gastroenterology and Internal Medicine, Postgraduate Institute of Medical Education and Research, Chandigarh, India

Histoplasma capsulatum is the causative organism for histoplasmosis. Usually transmitted through inhalation of spores, the disease primarily involves lung. However, in immunocompromised individuals it may disseminate throughout the body involving various organs. Though GI involvement in disseminated histoplasmosis could present in up to $70 \%-80 \%$ of patients, symptomatic involvement is much less common and is reported in only $3 \%$ to $12 \%$ of patients. ${ }^{1,2}$ Primary GI involvement of histoplasmosis is still rarer and it is suspected to be spread by food or water ingestion. Ileum is the most common site of involvement of GI histoplasmosis (GIH) due to abundance of lymphatic and Peyer's patches. GIH can mimic intestinal tuberculosis and CD clinically and can have myriad of presentations. So, high clinical suspicion and early diagnosis is essential for better outcome. ${ }^{2,3}$

We report about a series of patients with GIH diagnosed in a gastroenterology unit in tertiary care center in North India over last 1 year. Data regarding mode of presentation, endoscopic and histological findings were retrieved. Diagnosis was established on histology by H\&E and Periodic Acid-Schiff (PAS) stain. Chest radiographs and computed tomographic images were reviewed. Risk factors for GIH were assessed in each case. Informed consent to publish was taken from the patients.

Received July 31, 2018. Revised August 6, 2018. Accepted August 7, 2018. Correspondence to Vishal Sharma, Department of Gastroenterology, Postgraduate Institute of Medical Education and Research, Sector 12, Chandigarh 160012, India. Tel: +91-708-7008099, Fax: +91-172-2744401, E-mail: docvishalsharma@gmail.com

ORCID Harshal S Mandavdhare (https://orcid.org/0000-0001-8020-9848), Vishal Sharma (https://orcid.org/0000-0003-2472-3409)
Six patients with GIH were diagnosed over 1 year. Mean age was $43 \pm 13.08$ years (range, 25-60 years) and 3 patients (50\%) were male. The presenting complaints in decreasing order were abdominal pain ( 5 patients), weight loss ( 5 patients), fever (4 patients), diarrhea (2 patients), dysphagia (2 patients) and hematochezia (1 patient).

Two patients had HIV as a risk factor for GIH but none were previously diagnosed. One patient had history of gradually increasing dysphagia for last 6 months for which upper GI endoscopy was done which revealed presence of multiple deep ulcers in upper esophagus (Fig. 1A) and CT thorax showed long segment mild asymmetric circumferential thickening of mid-esophagus with multiple variable sized nodules in both lobes of lung and the histology was consistent with histoplasmosis (Fig. 1B). Other patient had intermittent colicky abdominal pain, for which contrast-enhanced CT (CECT) abdomen was done which revealed presence of circumferential mural thickening of terminal ileum, ileocecal (IC) junction and cecum. Colonoscopy showed circumferential ulcers in cecum. On evaluation they have been diagnosed with HIV with low CD4 counts (143 and 125 cells $/ \mathrm{mm}^{3}$ respectively). Neither of the patient was on highly active antiretroviral therapy.

The third patient had presented with abdominal pain, weight loss and bloody diarrhea over last 6 months. On CECT abdomen there was asymmetric mural thickening in terminal ileum and IC junction with dilatation of terminal ileum along with thickening of antropyloric region. On endoscopy patient had a large ulcero-proliferative growth near IC valve and nonnegotiable growth near antrum. Histology confirmed the pres- 

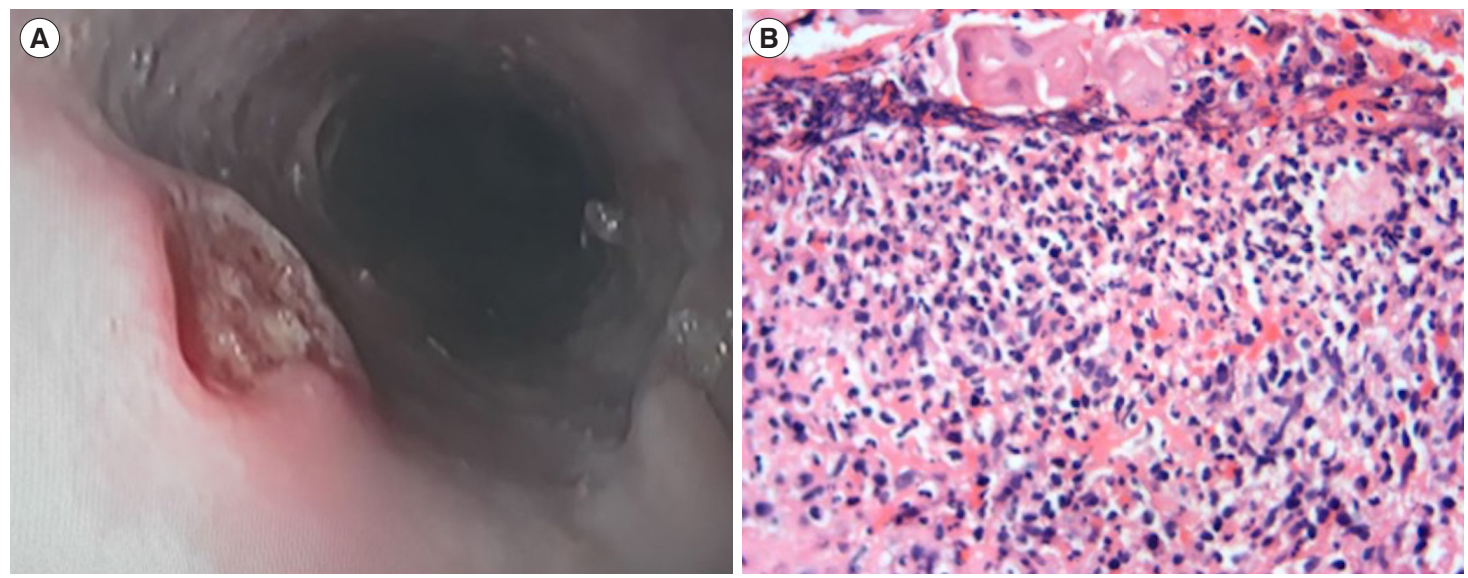

Fig. 1. (A) Esophageal circular deep ulcer and (B) histology showing Histoplasma $\left(H \& E_{1} \times 40\right)$.
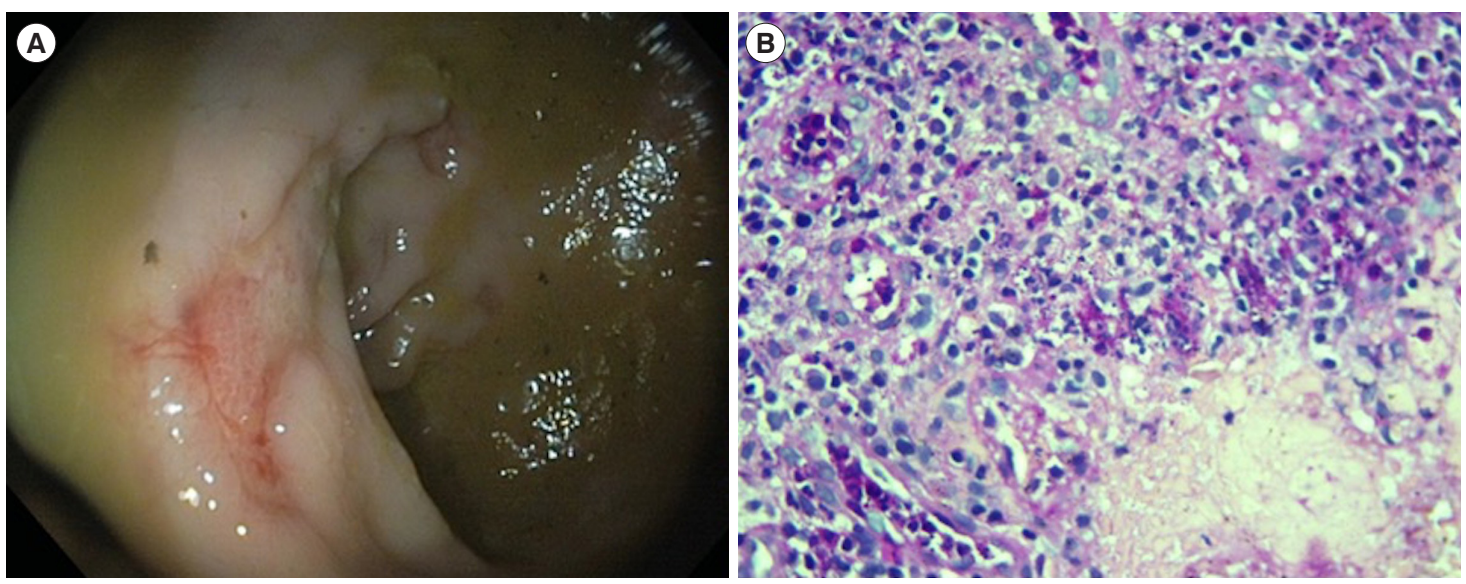

Fig. 2. (A) Rectal ulcer seen on sigmoidoscopy and (B) histology showing multiple tiny intracellular and extracellular organisms (Periodic acid Schiff stain, $\times 40)$.

ence of diffuse large B cell lymphoma. Fourth patient presented with abdominal pain, fever and diarrhea for 3 months. CECT chest and abdomen revealed multiple coalescing nodules in both the lungs with multiple enlarged necrotic retroperitoneal, mesenteric, prevascular and paratracheal lymph nodes. Fineneedle aspiration of this lymph node was AFB positive. Upper GI endoscopy was done for mild dysphagia which showed mid esophagus large irregular ulcer with surrounding hyperemia, histopathological examination of which revealed findings suggestive of histoplasmosis. The 5 th patient presented with history of abdominal pain, fever and diarrhea for 2 weeks. Patient was a known case of diabetes mellitus for last 4 years which was poorly controlled with glycosylated hemoglobin of 11.5 and patient was noncompliant to the medications. Sigmoidoscopy showed multiple ulcers (Fig. 2) of varying sizes in rectum and histology confirmed the diagnosis. Sixth patient was a lady with intermittent colicky abdominal pain, fever and weight loss for 1 year. On CECT abdomen there was thickening of cecum, IC junction and terminal ileum with surrounding inflammatory changes. Colonoscopy was performed which revealed growth in cecum with gaping of ileo-cecal valve and ulcers in terminal ileum. Histological examination revealed findings which were suggestive of histoplasmosis. On risk factor evaluation the patient was found to have idiopathic CD4 lymphocytopenia ( 16 cells $/ \mathrm{mm}^{3}$ ). However, T and B lymphocyte counts and Ig profile was normal.

Diagnosis was confirmed in each case by histological examination which revealed presence of infiltration by acute and chronic inflammatory cells including macrophages and histiocytes. PAS staining revealed presence of multiple intracellular as well as extracellular small organisms ranging in size from 2 to $3 \mu \mathrm{m}$ with eccentrically placed nuclei and peripheral halo suggestive of Histoplasma. None of the patient had presence of granuloma on histological analysis. 
All patients received injectable amphotericin B for 2 weeks followed by oral itraconazole $200 \mathrm{mg}$ BD apart from treatment for primary disease. All patients responded well with the treatment. Follow-up endoscopy was done after 4 weeks of treatment in 4 patients. Follow-up endoscopy revealed healing of mucosal lesions in the esophagus and ileo-colonic area in 2 patients each.

Histoplasmosis was first reported in India in 1954. Most cases occurring in West Bengal and Assam states. ${ }^{4}$ However, it is being underdiagnosed in India possibly due to high prevalence of tuberculosis. Patients usually presents with non-specific complains like abdominal pain, anorexia, weight loss, diarrhea and hematochezia. Bowel obstruction and perforation due to GIH is also reported. ${ }^{2,3,5}$ These nonspecific presentations are associated with delay in diagnosis and poor outcome. In our case series also patients had presented with nonspecific complains like abdominal pain, weight loss, fever, diarrhea, and so forth. However, high clinical suspicion and early diagnosis with treatment had improved outcome in our patients. Esophageal involvement in GIH can be either due to mediastinal adenitis in which inflamed mediastinal lymph nodes impinge on esophagus causing ulceration and hyperemia or due to fibrosing mediastinitis which is due to exuberant fibrous reaction secondary to H. capsulatum causing compression of various mediastinal structures including esophagus. ${ }^{6}$ In our case series 2 patients had esophageal involvement with presence of ulcers in esophagus. Both the patients had multiple coalescing nodules in both the lungs raising suspicion that esophageal involvement might be secondary to the lung involvement.

Although disseminated histoplasmosis can involve any part of intestine, ileum is the most commonly affected area due to abundance of lymphoid tissue and Payers patches. In our case series ileum (2 patients) and cecum (1 patient) were the most commonly involved areas. GIH usually produces bowel wall thickening, mass lesion, lymphadenopathy, signs of small bowel obstruction and free peritoneal air. In our case series all patients had radiological signs with thickening of involved bowel wall, proximal dilation of the small bowel and local lymphadenopathy. Patients with esophageal histoplasmosis also showed presence of scattered nodules in both the lungs. Endoscopically GIH may present with focal or multiple ulcerations, erythema, large inflammatory mass, annular constricting lesion or polypoidal mass involving any part in intestine. ${ }^{5}$ In our series both the patients with esophageal involvement had ulceration with erythema in esophagus. On colonoscopy 2 patients had proliferative growth and 2 patients had ulcerations.
It is well recognized that disseminated disease is usually present in immunocompromised individuals. Moreover, immunosuppressed patients present with atypical clinical features hindering diagnosis and treatment. In our case series we had 2 patients of acquired immune deficiency syndrome (AIDS) with low CD4 counts (143 and 125 cells $/ \mathrm{mm}^{3}$ ) and 1 patient each of primary GI lymphoma, disseminated tuberculosis with severe malnutrition, isolated low CD4 counts (16 cells/ $\mathrm{mm}^{3}$ ) and diabetes mellitus.

Diagnosis of histoplasmosis can be established by microscopic examination, antigen testing, serological testing, culture and molecular techniques. H. capsulatum appears as small, 2 to $4 \mu \mathrm{m}$ in diameter, ovoid to spherical in shape. They are usually found in cytoplasm of macrophages with "halo-like" zone of clear area around the organism. ${ }^{7}$ In our study diagnosis was established by histological examination with presence of multiple intracellular as well as extracellular small organisms ranging in size from 2 to $3 \mu \mathrm{m}$ with eccentrically placed nuclei and peripheral halo.

Treatment of severe or disseminated histoplasmosis involves initial therapy with amphotericin B for 2 weeks followed by oral itraconazole $200 \mathrm{mg}$ BD for 3 to 6 months depending on response and patients condition. ${ }^{8}$ In AIDS patients itraconazole is usually continued till CD4 counts increases more than 150 cells $/ \mathrm{mm}^{3}$. In mild or less severe cases oral itraconazole therapy alone can also be sufficient. Prognosis is poor in patients with disseminated histoplasmosis with mortality up to $25 \%$ even with treatment with amphotericin B. ${ }^{9}$ In our study we had treated all patients with amphotericin B for 2 weeks followed by oral itraconazole therapy which was associated with good outcome without any mortality in our study. We had also repeated endoscopy to document endoscopic response in 4 patients after 4 weeks of therapy.

To conclude, GIH is not uncommon even in countries endemic with tuberculosis like India. High clinical suspicion in presence of immunocompromised state and differentiating it from tuberculosis or $\mathrm{CD}$ is associated with early diagnoses. Vigilant search for risk factors should be done in each case. Finally, aggressive and prompt treatment of GIH is associated with good outcome.

\section{FINANCIAL SUPPORT}

The authors received no financial support for the research, authorship, and/or publication of this article. 


\section{CONFLICT OF INTEREST}

No potential conflict of interest relevant to this article was reported.

\section{AUTHOR CONTRIBUTION}

Conceptualization: Mandavdhare HS and Sharma V. Writing, original draft: Mandavdhare HS and Shah J. Critical revision for intellectual content: Suri V, Kumari S, Dutta U, and Sharma V. Patient care: Mandavdhare HS, Prasad KK, Suri V, Kumari S, Dutta U, and Sharma V. Histology: Prasad KK. Manuscript draft: Agarwala R. Final approval to manuscript: all authors

\section{REFERENCES}

1. Wheat LJ, Connolly-Stringfield PA, Baker RL, et al. Disseminated histoplasmosis in the acquired immune deficiency syndrome: clinical findings, diagnosis and treatment, and review of the literature. Medicine (Baltimore) 1990;69:361-374.

2. Suh KN, Anekthananon T, Mariuz PR. Gastrointestinal histoplasmosis in patients with AIDS: case report and review. Clin Infect Dis 2001;32:483-491.
3. Lamps LW, Molina CP, West AB, Haggitt RC, Scott MA. The pathologic spectrum of gastrointestinal and hepatic histoplasmosis. Am J Clin Pathol 2000;1 13:64-72.

4. Panja G, Sen S. A unique case of histoplasmosis. J Indian Med Assoc 1954;23:257-258.

5. Spivak H, Schlasinger MH, Tabanda-Lichauco R, Ferstenberg H. Small bowel obstruction from gastrointestinal histoplasmosis in acquired immune deficiency syndrome. Am Surg 1996;62:369-372.

6. Kefri M, Dyke S, Copeland S, Morgan CV Jr, Mehta JB. Hemoptysis and hematemesis due to a broncholith: granulomatous mediastinitis. South Med J 1996;89:243-245.

7. Edwards MR, Hazen EL, Edwards GA. The micromorphology of the tuberculate spores of Histoplasma capsulatum. Can J Microbiol 1960;6:65-70

8. Wheat LJ, Freifeld AG, Kleiman MB, et al. Clinical practice guidelines for the management of patients with histoplasmosis: 2007 update by the Infectious Diseases Society of America. Clin Infect Dis 2007;45:807-825.

9. Johnson PC, Wheat LJ, Cloud GA, et al. Safety and efficacy of liposomal amphotericin B compared with conventional amphotericin B for induction therapy of histoplasmosis in patients with AIDS. Ann Intern Med 2002;137:105-109. 